\title{
ESTUDANDO A RENDA DO TRABALHO DA POPULAÇÃO RURAL: O CASO DE PALMITOS, SC
}

\author{
Studying the work income of rural population: the case of Palmitos \\ hearts, SC
}

\section{El estudio de los ingresos del trabajo de la población rural: el caso de Palmitos, SC}

\author{
Luiz Panhoca ${ }^{1}$ \\ Olga Maria Panhoca da Silva²
}

\begin{abstract}
RESUMO
Este artigo apresenta uma análise do padrão de renda do trabalho a partir de uma análise do Índice de Desenvolvimento Humano - IDH. Aborda-se o problema da renda do trabalho e se mostram as dificuldades do equacionamento das políticas globais em virtude das diferenças regionais. Foi realizado um estudo transversal de base populacional no município de Palmitos, na região oeste do estado de Santa Catarina, Brasil. Aborda-se de forma critica a conceituação do espaço rural e urbano e lançam-se luzes sobre a contabilização da renda e sua equivocada leitura da divisão entre cidade e campo. Dentre as conclusões uma é que se deve sempre se ater ao fato de que a ação rural é diferente da ação urbana. Na zona rural a erradicação da pobreza não é suficiente por si, é preciso garantir a dignidade de viver com trabalho na sua própria terra, reunir a família em torno de sua própria mesa e se ter estabilidade de trabalho que garanta as necessidades básicas.

Palavras-chave: trabalho e renda; população rural; renda rural.
\end{abstract}

\begin{abstract}
This paper presents an analysis of the pattern of labor income from an analysis of the Human Development Index - HDI. Addresses the problem of labor income and show the difficulties of addressing global policy from a regional differences analysis. We conducted a population-based cross-sectional study in the city of Palmitos, in the western state of Santa Catarina, Brazil. It approaches a critical conceptualization of rural and urban to put lights on the accounting income and its mistaken reading of the division between town and country. Among the findings is that one should always stick to the fact that the rural action is different from the urban action. In rural poverty eradication is not enough by itself; we must ensure the dignity to live in work in their own land, gather the family around their own table and to have job stability that guarantees the basic necessities.

Keywords: work and income; rural population; rural incomes.
\end{abstract}

\section{RESUMEN}

Este artículo presenta un análisis de la estructura de los ingresos laborales a partir de un análisis de lo Índice de Desarrollo Humano - IDH. Aborda el problema de las rentas del trabajo y mostra los entraves de abordar la política mundial debido a las diferencias regionales. Se realizó un estudio poblacional de corte

1 Professor Adjunto de Contabilidade da UFPR, membro da ITCP/UFPR. E-mail: panhoca@ufpr.br

2 Pós Doutoranda em Geografia do Setor e Ciencias da Terra da UFPR. 
transversal en la ciudad de Palmitos, en Santa Catarina, Brasil. Se acerca a una concepción crítica de las zonas rurales y urbanas y se ponen luces en la contabilidad de los ingresos y de su lectura equivocada de la división entre campo y ciudad. Entre los hallazgos es que siempre debemos apegarse atencion al hecho de que es diferente la acción urbana de la acción rural. La erradicación de la pobreza rural no es suficiente por sí misma, debemos velar por la dignidad de vivir en el trabajo en su propia tierra, a la familia alrededor de su propia mesa y tener estabilidad laboral que garantiza las necesidades básicas.

Palabras clave: trabajo y ingresos; población rural; ingresos rurales.

\section{Agradecimentos}

Agradecemos de modo especial às famílias residentes no município de Palmitos que nos receberam em suas casas e que responderam aos questionários de forma paciente e gratuita; o apoio incondicional e a amizade de todos os membros do Grupo de estudos em Epidemiologia; ao Prof. Mário Cesar Nascimento agradecemos a amizade, a bondade, o companheirismo e o seu desprendimento para me ajudar; a fraternidade do Dr. Lauro Brito de Almeida do PPG Mestrado em Contabilidade do Setor de Ciências Sociais Aplicadas da UFPR por ter me recebido, $e$ acolhido o grupo de estudos de braços abertos, me deixando tranquila e motivada para trabalhar; à Agência IBGE/Palmitos, agradeço a boa vontade, simpatia e apoio. As Rádio Entrerios AM, Rádio 101.5 FM e ao Jornal Correio do Oeste agradeço pela colaboração na divulgação do nosso trabalho, assim como o presente apoio da Prefeitura de Palmitos e da 29a Secretaria de Desenvolvimento Regional do Estado de Santa Catarina que nunca nos deixou sem resposta; à coordenadora desse projeto na FAPESC, Fernanda Antonielle, que por sua disponibilidade e delicadeza aliada à competência, manteve o trabalho em andamento e possibilitou a sua conclusão.

\section{Introdução}

Um dos problemas brasileiros a serem equacionados é o padrão de renda do trabalho da população que se apresenta de modo peculiar para cada região. A parte sul do Brasil, apesar de um IDH considerado bom, tem a renda do trabalho como um dos entraves para o pleno desenvolvimento. A diferenciação entre a população rural e urbana, e entre a população litorânea e interiorana é uma das dificuldades em se obter resultados favoráveis na aplicação e na elaboração de projetos que impulsionem o incremento de renda do tralho. Para enfrentar esse quadro é necessário se entender a renda do trabalho sob ponto de vista regional. Veiga (2001) critica a conceituação do espaço rural e urbano e lança luzes sobre a contabilização da renda e sua equivocada leitura da divisão cidade e campo. Este trabalho teve como objetivo estudar as pequenas comunidades do extremo oeste do estado de Santa Catarina sob a visão de José Eli da Veiga. Optou-se pelo município de Palmitos, estado de Santa Catarina, dado o acelerado processo de envelhecimento da população, sua vocação para a agricultura e por sua vida rural. Estes fatores dão a oportunidade da identificação do trabalho e do comportamento de uma população rural do sul do Brasil.

\section{Método}

\section{Delineamento do estudo}

Foi realizado um estudo transversal de base populacional tendo como referência para a coleta de dados o dia 20 de julho de 2008 . A população alvo do estudo foram os domicílios do município de Palmitos, localizado na região oeste do estado de Santa Catarina. Os 
inquéritos de base populacional são de extrema importância para a identificação dos fenômenos populacionais como renda, educação e saúde, e fornecem informações para o planejamento das ações locais e para o monitoramento das comunidades e grupos de pessoas vulneráveis (BRASIL, 2004, p. 136).

Para a coleta de dados seguiu-se a proposta da pesquisa do "Inquérito domiciliar sobre comportamentos de risco e morbidade referida de doenças e agravos não transmissíveis, Brasil, 15 capitais e Distrito Federal, 2002-2003" (BRASIL, 2004). A amostragem foi por conglomerados autoponderada em dois estágios de seleção, sendo as unidades primárias os setores censitários e as unidades secundárias os domicílios (SZWARCWALD; DAMACENA, 2008). A seleção dos setores foi de forma sistemática, com probabilidade de seleção proporcional ao número de domicílios que os mesmos possuíam por ocasião do censo demográfico de 2007 (SILVA, 2003).

O município de Palmitos possuía 36 setores censitários urbanos e 14 setores rurais, que foram sorteados resultando em 14 setores rurais e 28 setores urbanos a serem visitados (LEBRÃO; DUARTE, 2003; DUARTE, 2001). Os domicílios foram sorteados de modo sistemático dentro dos setores escolhidos e agrupados em segmentos de três domicílios consecutivos. Foram visitados os domicílios sorteados e, aceitos para inclusão no estudo, os considerados particulares e ocupados de modo permanente.

A classificação de rural e urbano segue a classificação do IBGE. Esta classificação é determinada pela Câmara Municipal local e segue critérios políticos.

$\mathrm{O}$ modelo de questionário usado encontra-se no endereço eletrônico do INCA $<$ www.inca.org.br $>$. Para este estudo utilizou-se o questionário domiciliar que é composto por perguntas de aspectos demográficos e socioeconômicos.
Foi divulgado nas rádios locais e no jornal da cidade a data do início das entrevistas, assim como o objetivo e método da pesquisa. Durante o estudo foi mantida a privacidade dos indivíduos, garantindo a participação anônima e voluntária. Utilizou-se um Termo de Consentimento Livre e Esclarecido, para cada pessoa entrevistada. Os entrevistados assinaram os termos como uma condição necessária para a participação no estudo, e os critérios foram definidos pelo Comitê de Ética em Pesquisa (CEP) em Seres Humanos da Universidade do Estado de Santa Catarina (UDESC).

Para controlar a qualidade, o trabalho de campo contou com: (i) o mapeamento da área com treinamento oferecido pela Coordenadora da Agência IBGE de Palmitos - SC; (ii) mapeamento dos domicílios a serem entrevistados na zona rural e urbana para identificação dos domicílios, contato prévio com os moradores e elaboração do banco de dados de endereços rurais.

\section{Para a análise}

Foram usadas a renda familiar, a renda per capita, a pobreza, o índice de Gini e a curva de Lorenz.

A Renda familiar foi considerada a renda relatada. A renda per capita é a razão entre o somatório da renda pessoal dos indivíduos do domicilio e o número total destes. Os valores relativizados em salários mínimos estão expressos nos do ano de 2008, com valor de $\mathrm{R} \$ 415,00$.

A pobreza é definida como a proporção dos indivíduos com renda familiar ${ }^{3}$ per capita ${ }^{4}$ inferior a 50\% do salário mínimo (UN, 2006).

O Índice de Gini mostra a desigualdade da renda (de zero a um), em uma população onde o valor zero corresponde à igualdade $3 \quad$ A unidade de análise neste trabalho é o domicílio e não a família. A utilização do domicílio como unidade de análise é o que mais se aproxima do conceito de unidade orçamentária utilizado na PNAD.

4 Rendimento mensal familiar per capita é a soma dos rendimentos dos componentes da família, exclusive empregados e pensionistas dividida pelo número de membros da família (IBGE, 2010). 
perfeita. É calculado a partir da renda sendo a razão do montante dos $40 \%$ mais pobres e dos $10 \%$ mais ricos (2007).

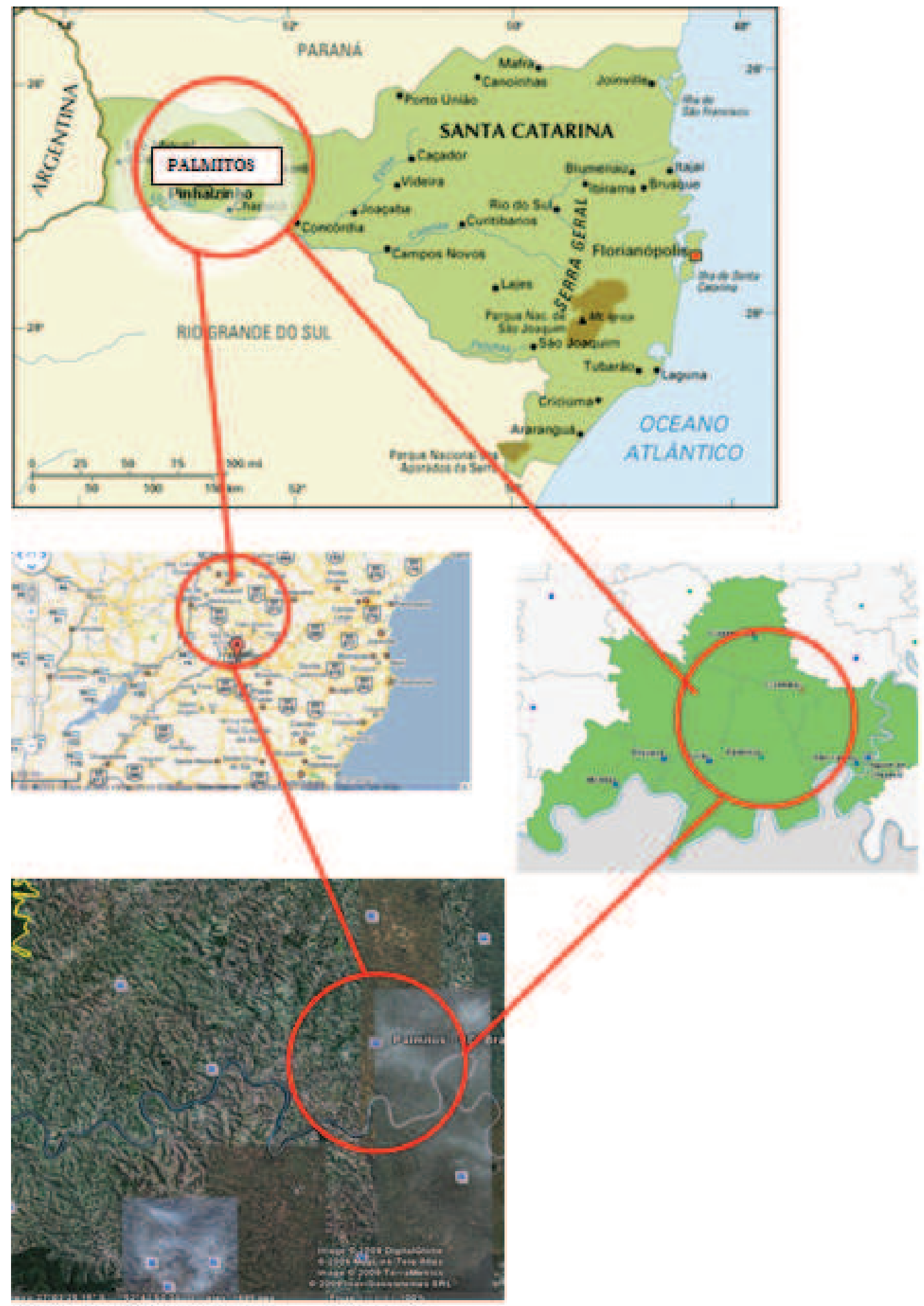

FIGURA 1 - LOCALIZAÇÃO DO MUNICÍPIO DE PALMITOS 
A curva de Lorenz foi desenvolvida pelo economista Max O. Lorenz para representar a distribuição relativa de uma variável em um domínio determinado. O domínio, por exemplo, pode ser o conjunto de pessoas de uma região ou país. A variável cuja distribuição se estuda pode ser a renda dessas pessoas. A curva é traçada considerando-se a percentagem acumulada de pessoas no eixo das abscissas e a percentagem acumulada de renda no eixo das ordenadas. Neste estudo, a curva de Lorenz mostra a classificação escalar do posicionamento relativo dos indivíduos da população na participação na renda total. Para uma distribuição perfeitamente igualitária, a curva de Lorenz é uma linha reta e corresponde à "igualdade".

TABELA 1 - DISTRIBUIÇÃO DOS MORADORES POR DOMICILIO PARAA ZONA RURAL E URBANA
16.061 habitantes com a densidade populacional de 45,7 habitantes por $\mathrm{Km}^{2}$.

O município de Palmitos, que é composto por quatro distritos, compondo 34 setores censitários subdivididos em rural e urbano. Dos 160 domicílios sorteados, encontraram-se 156 domicilios. As casas de madeira são comuns nessa região, elas podem ser transportadas para outra localidade quando a familia muda de endereço ou simplesmente são vendidas para outras pessoas que as transportam para outro local. Às vezes, são queimadas ou desmanchadas para se reaproveitar a madeira e facilmente desaparecem os vestígios da habitação. Dos 156 domicilios encontrados, 136 foram entrevistados, uma vez que alguns domicilios encontravam-se fechados, estavam vagos na data da entrevista ou recusaram a entrevista.

Notou-se que dos domicílios que esta

\begin{tabular}{c|c|c|c|c|c|c}
\hline & \multicolumn{2}{|c|}{ Rural } & \multicolumn{2}{c|}{ Urbano } & \multicolumn{2}{c}{ TOTAL } \\
\hline $\begin{array}{c}\text { Número de moradores por } \\
\text { domicílio }\end{array}$ & Pessoas & Domic. & Pessoas & Domic & Pessoas & Domic \\
\hline 2 & 44 & 22 & 32 & 15 & 76 & 37 \\
3 & 63 & 21 & 36 & 12 & 99 & 33 \\
4 & 68 & 17 & 48 & 12 & 116 & 29 \\
5 & 40 & 8 & 45 & 9 & 85 & 17 \\
\hline 1 & 3 & 3 & 7 & 7 & 10 & 10 \\
6 & 24 & 4 & 6 & 1 & 30 & 5 \\
7 & 14 & 2 & 7 & 1 & 21 & 3 \\
8 & 8 & 1 & 8 & 1 & 16 & 2 \\
\hline TOTAL & 264 & 78 & 189 & 58 & 451 & 136 \\
\hline
\end{tabular}

vam nestas condições, 6 negaram a participação (todos da zona urbana, e que nos domicílios

Resultados

O município de Palmitos, Figura 1, ocupa uma área de $351 \mathrm{Km}^{2}$ e está situado na região oeste do estado de Santa Catarina fazendo fronteira o Estado do Rio Grande do Sul. O PIB per capita para o ano de 2006 foi de $\mathrm{R} \$ 15.026,00$

com incidência de pobreza em 23,9\% e índice de Gini igual a 0,35 para o ano de 2003 (IBGE, 2007b). De acordo com a contagem populacional de 2007 (IBGE, 2007a), a população era de encontrados fechados, seus habitantes estavam trabalhando nas terras da zona rural.

O total de pessoas entrevistadas foi de 453, mas quando classificados os setores, a zona rural apresentou 264 indivíduos em 78 domicílios e a urbana, 189 pessoas e 58 domicílios, Tabela 1. A média de pessoas por domicilio na zona rural foi de 3,4 pessoas por domicílios e na zona urbana foi de 3,2.

A renda média da população é de $\mathrm{R} \$ 1.980,90$ e $\mathrm{R} \$ 1.999,57$, respectivamente para a população residente na zona rural e urbana. 
Na Figura 2, pode-se observar que a maioria das familias tem renda de até seis salários mínimos, tanto as familias rurais como as urbanas, sendo que a maior concentração de familias aparece para dois salários mínimos. O percentual de pobres é de $21,3 \%$ da população rural e $24,0 \%$ da urbana.
Cabe destacar, observando a curva de Lorenz, Figura 3, que não existe diferença na distribuição da renda entre a população rural e urbana, onde ambas as curvas quase se sobrepõem. No entanto elas se apresentam abaixo da curva de igualdade enfatizando que essa distribuição de renda está concentrada em uma

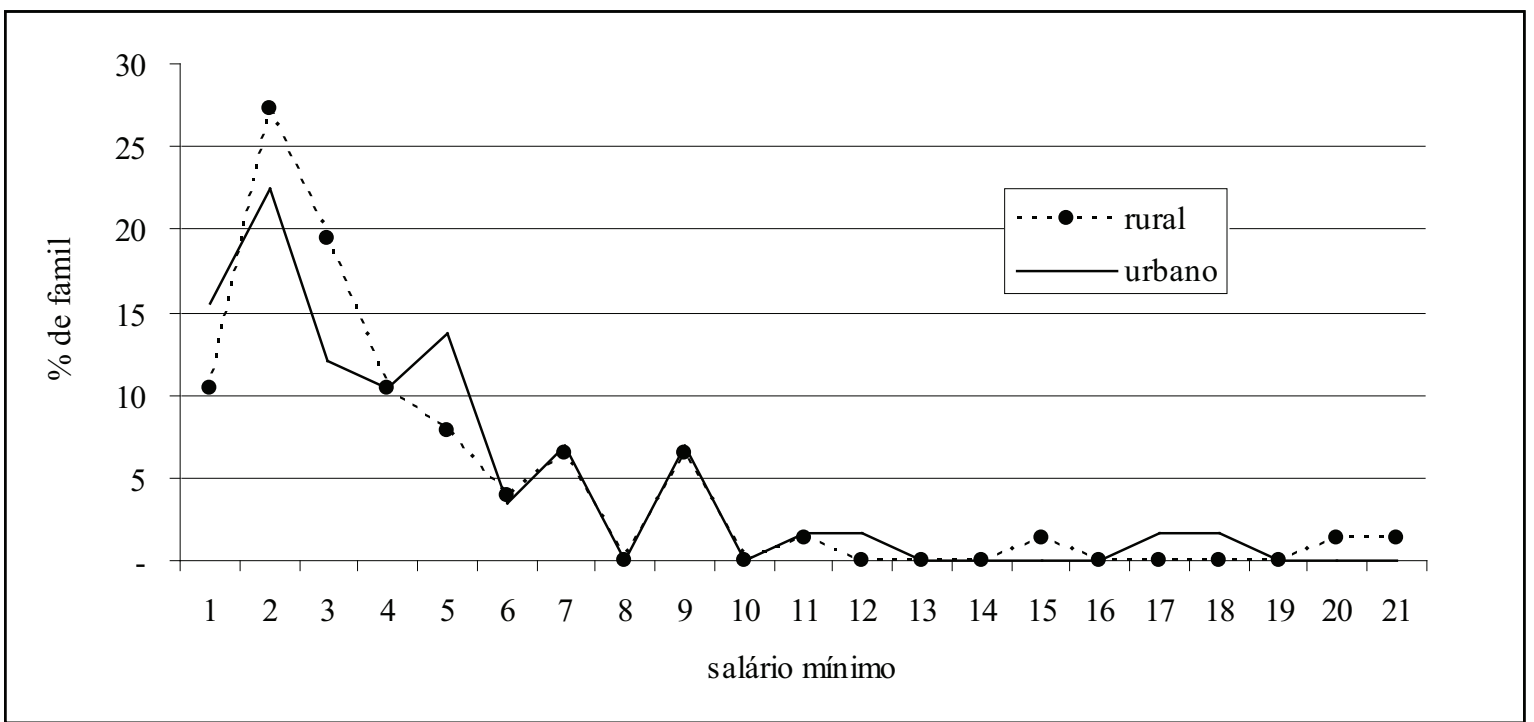

FIGURA 2 - DISTRIBUIČ̃̃O PERCENTUAL DAS FAMÍLIAS EM RELAÇÃO À RENDA FAMILIAR (EM SALÁRIOS MÍNIMOS), PALMITOS, SC, 2008.

O Índice de Gini é 0,30 para a população rural e 0.29 para a urbana, uma vez que, tanto para a população da zona rural quanto para a urbana, os $40 \%$ mais pobres recebem $12 \%$ da renda dessa população, enquanto que os $10 \%$ mais ricos da zona rural ficam com $42 \%$ da renda e no caso da zona urbana os $10 \%$ mais ricos ficam com $39 \%$ da renda. parcela pequena da população. A curva mostra que $50 \%$ das pessoas mais pobres da população ficam com apenas $18 \%$ da renda, ao passo que $15 \%$ dos mais ricos ficam com os outros $50 \%$ da renda. Pode-se afirmar inequivocamente por qualquer indicador de desigualdade utilizado que essa distribuição não é equitativa.

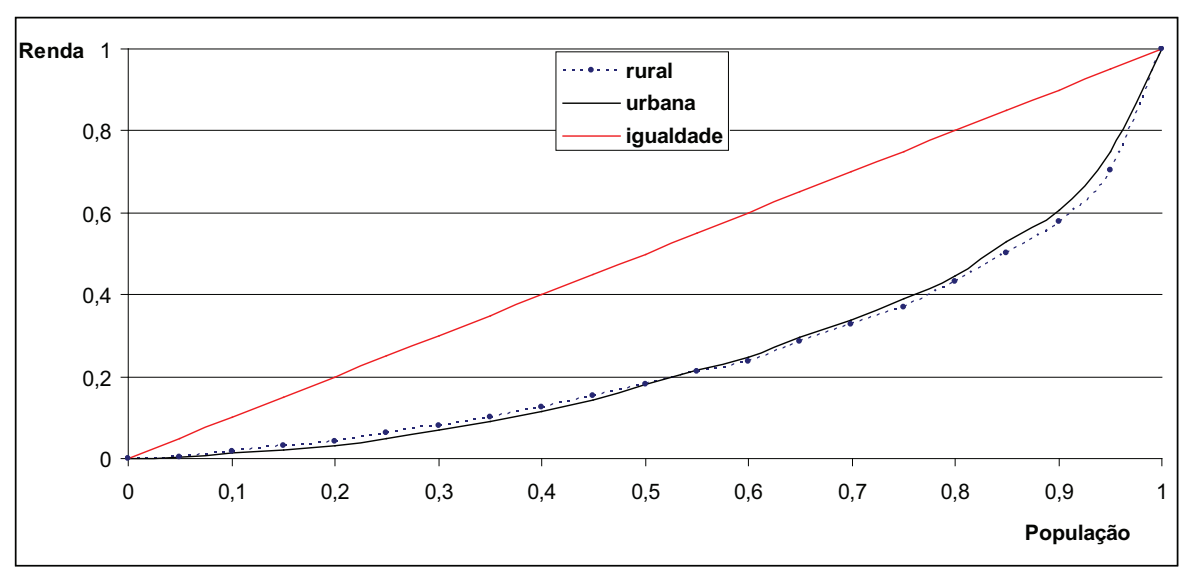

FIGURA 3 - CURVA DE LORENTZ PARA A RENDA DA POPULAÇÃO RURAL E URBANA DE PALMITOS, SC, 2008. 
O hiato da pobreza, entendido como a distância da renda da pessoa pobre $(\mathrm{Y})$ até meio salário mínimo (Z) foi calculado como: Z-Y/Z. ponto de vista regional. José Eli da Veiga critica a conceituação do espaço rural e urbano e lança luzes sobre a contabilização da renda e sua equivocada leitura da divisão cidade e campo.

\section{Conclusão}

Nunca se pode perder de vista que qualquer emancipação da pobreza não basta por si, é preciso ter duas coisas em mente: "A primeira é a dignidade de viver com trabalho na sua própria terra, e reunir a família em torno de sua própria mesa. A segunda é ter estabilidade de trabalho que garanta as "necessidades básicas" (VEIGA, 2002a).

Segundo Veiga (2001, p. 101), "um dos maiores obstáculos à renovação das idéias sobre o desenvolvimento da sociedade brasileira é a força que adquiriu nas últimas décadas o mito de sua avassaladora urbanização" tem-se a impressão que habitamos um país urbano, causando vieses de análises e nas políticas públicas.

A metodologia adotada por Veiga para definir "rural" e "urbano" mostra-nos que ainda existe espaços para pesquisa e estudos mais aprofundados. Continuando a linha de raciocínio do mesmo autor, questiona-se ainda se "seriam os ecossistemas mais alterados pela ação humana, as manchas ocupadas por megalópoles e a pressão antrópica" a "[...] artificialização dos ecossistemas" o efetivo indicador do grau de urbanização dos territórios (VEIGA, 2002a, p. 33).

Constata-se, portanto, que as argumentações de Eli da Veiga fazem sentido na medida em que o padrão da renda do trabalho da população se apresenta de modo peculiar para a região estudada. A região estudada, apesar de um "bom" IDH, tem na renda e na diferenciação entre a população rural e urbana, uma das dificuldades em se obter resultados favoráveis na aplicação e na elaboração de projetos que impulsionem o incremento de renda. Para enfrentar esse quadro é necessário se entender a renda do
BRASIL. Ministério da Saúde. Secretaria de Vigilância em Saúde. Secretaria de Atenção à Saúde. Instituto Nacional de Câncer (INCA). Coordenação de Prevenção e Vigilância. Inquérito domiciliar sobre comportamentos de risco e morbidade referida de doenças e agravos não transmissíveis: Brasil, 15 capitais e Distrito Federal. 20022003. Rio de Janeiro: INCA, 2004.

DATAFOLHA. Sessenta por cento dos brasileiros estão parados. Folha de São Paulo, São Paulo, 25075 ed. 27 nov. 1997. Caderno Especial: Mexa-se.

DUARTE, Y.A.O. Família-rede de suporte ou fator estressor: a ótica de idosos e cuidadores familiares. Tese (Doutorado) - Escola de Enfermagem, Universidade de São Paulo, São Paulo, 2001.

INSTITUTO BRASILEIRO DE GEOGRAFIA E ESTATÍSTICA (IBGE). Pesquisa nacional por amostra de domicílios: Brasil, 2006. Rio de Janeiro: IBGE, v. 27, 2007a. 123 p.

Estatísticas do século XX: Conceitos e definições. Disponível em: <www.ibge.gov.br/seculoxx/anexos/ conceitos.pdf - 2009-07-20> - Text Version. Acesso em: $15 / 5 / 2010$, s.d.

Desigualdade de renda no Brasil : uma análise da queda recente / organizadores: Ricardo Paes de Barros, Miguel Nathan Foguel, Gabriel Ulyssea. - Brasília: Ipea, 2007b. 2 v.

LEBRÃO ML, DUARTE YAO. SABE - Saúde, Bem-Estar e Envelhecimento. O projeto SABE no Município de São Paulo: uma abordagem inicial. Brasília: Organização PanAmericana da Saúde; 2003.

IPEA. Definição e metodologia de cálculo dos indicadores $e$ índices de desenvolvimento humano e condições de vida. In: Metodologia utilizada pelas equipes da Fundação João Pinheiro (FJP) e do Instituto de Pesquisas Econômicas Aplicadas (IPEA), na construção dos indicadores e índices publicados no Atlas de Desenvolvimento Humano no Brasil, 1.998

PEREIRA, J. C. R. Análise de dados qualitativos: estratégias metodológicas para as ciências da saúde humana e sociais. 3 ed. São Paulo: Edusp, 2001.

PERES, M. A.; TRAEBER, J; MARCENES, W. Calibração de examinadores para estudos epidemiológicos de cárie 
dentária. Cadernos de Saúde Pública, v. 17, n. 1, p. 153159, jan./fev. 2001.

PINHO, A. A.; FRANCA-JUNIOR, I. Prevenção do câncer de colo do útero: um modelo teórico para analisar o acesso e a utilização do teste de Papanicolau. Revista Brasileira de Saúde Materna e Infantil, Recife, v. 3, n. 1, mar. 2003.

PNUD. Programa das Nações Unidas para o Desenvolvimento. Objetivos de desenvolvimento do milênio. Disponível em: <www.pnud.org.br/odn>. Acesso em: 31/01/2009.

REGO, A. L. V.; CHIARA, V. L. Nutrição e excesso de massa corporal: fatores de risco cardiovascular em adolescenes. Revista de Nutrição, Campinas, v. 19, n. 6, 2006.

REICHENHEIM, M. E.; WERNECK, G. L. Anos potenciais de vida perdidos no Rio de Janeiro, 1990. As mortes violentas em questão. Cadernos de Saúde Pública, Rio de Janeiro, v. 10, s. 1, p. 188-198, 2007.

RIBEIRO, R. C. A. construção de um município saudável: descentralização e intersetorialidade - experiência de Fortaleza. Saúde e Sociedade, São Paulo, v. 6, n. 2, dez. 1997.

ROCHA, N. S.; FLECK, M. P. A. Validade da versão brasileira do WHOQOL-BREF em pacientes deprimidos usando o modelo de Rasch. Revista de Saúde Pública, São Paulo, v. 43, n. 1, p. 147-153, 2009.

SEELIG, M. F; CAMPOS, C. R. J. de; CARVALHO, J. da C. A ventilação e a fumaça ambiental de cigarros. Ciência e Saúde Coletiva, Rio de Janeiro, v. 10, supl. 0, set./dez. 2005.

SILVA, N. N. Aspectos metodológicos: processo de amostragem. In: LEBRÃO, M. L.; DUARTE, Y. A. O. (Orgs.). O projeto SABE no município de São Paulo: uma abordagem inicial. Brasília: Organização Pan-Americana da Saúde, Ministério da Saúde; 2003. p. 47-57.

SILVA, O. M. P. O envolvimento da face na violência ocorrida na cidade de São Paulo ao longo de sua metropolização. São Paulo: Ed. Eletrônica da USP, 2001. p. 59. Disponível em: <www.bibcir.fsp.usp.br>. Acesso em: 10/7/2008.

SILVA, O. M. P.; SILVA, J. K. O.; REZENDE, R. A qualidade de vida na Zona Rural. A família Esperança. v. 1. Curitiba: Ed. O. M. P. SILVA. 2009. p. 46.

SOUZA, L. A.; COUTINHO, E. S. F. Fatores associados à qualidade de vida de pacientes com esquizofrenia. Revista Brasileira de Psiquiatria, São Paulo, v. 28, n. 1, 2006.

SZWARCWALD, C. L.; DAMACENA, G. N. Amostras complexas em inquéritos populacionais: planejamento e implicações na análise estatística dos dados. Revista
Brasileira de Epidemiologia, Sao Paulo, v. 11, supl. 1, p. 38-45, 2008.

UN - UNITED NATIONS. Annual World Report. UN, Washington DC. 2006.

U.S. DEPARTMENT OF HEALTH AND HUMAN SERVICES. (U. S.) Centers for Disease Control and Prevention (CDC). National Center for Chronic Disease Prevention and Health Promotion. Physical activity and health: A report of the surgeon general. Atlanta, GA: CDC, 1996. Disponível em: <www.cdc.gov/ nccdphp/sgr/>. Acesso em 27/12/2003.

UNERMAN, J.; BEBBINGTON, J.; O’DWYER, B. Sustainability accounting and accountability. New York: Routeledge, 2007.

VEIGA, J. E. O Brasil rural ainda não encontrou seu eixo de desenvolvimento. In: Estudos Avançados. Universidade de São Paulo. Instituto de Estudos Avançados. v. 15, n. 43. São Paulo: IEA, 2001.

Comments on "Access to land for rural poor". Econômica, v. 4, n. 2, dez. 2002a. p. 283-292.

. Cidades imaginárias: o Brasil é menos urbano do que se calcula. Campinas, SP: Autores Associados, 2002b.

WHO. WORLD HEALTH ORGANIZATION. Calibration of examiners for oral health epidemiology aurveys. Geneva: WHO, 1993. (Technical Report)

Promoting healthy life. Geneva: WHO, 2002a.

The world health report 2002: reducing the risks. Geneva: WHO, 2002b.

Recebido em: 31/out/2011

Aprovado em: 14/nov./2011 